\title{
Critical exponents of the chiral transition in strong coupling QCD
}

\author{
G. Boyd ${ }^{\text {a }}$, J. Fingberg ${ }^{\text {a }}$, F. Karsch ${ }^{\text {a,b }}$, L. Kärkkäinen ${ }^{\text {b }}$ and B. Petersson ${ }^{\text {b }}$ \\ ${ }^{a}$ HLRZ, c / o KFA Jülich, Postfach 1913, D-5170 Jülich, Germany \\ ${ }^{\mathrm{b}}$ Fakultät für Physik, Universität Bielefeld, D-4800 Bielefeld 1, Germany
}

Received 7 November 1991

(Revised 14 January 1992)

Accepted for publication 16 January 1992

\begin{abstract}
We study the critical behaviour of the chiral phase transition of SU(3) lattice QCD with one species of staggered fermions in the strong coupling limit. We find a second-order phase transition which seems to be controlled by an effective action which is in the same universality class as three-dimensional $O(2)$ spin models. In particular, we find for the exponent $\delta$, $0.18<1 / \delta<0.25$, in good agreement with the three-dimensional $\mathrm{O}(2)$ value, $1 / \delta=0.21$.
\end{abstract}

\section{Introduction}

During the last few years the critical behaviour of finite-temperature $\mathrm{SU}(N)$ gauge theories has been studied extensively (for a recent review see ref. [1]). It turned out that indeed these theories are in the same universality class as three-dimensional spin models with global $\mathrm{Z}(N)$ symmetry as originally suggested by Svetitsky and Yaffe [2]. In particular, results for the critical exponents of the SU(2) theory are in complete agreement with the exponents of the three-dimensional Ising model [3].

Compared to these high-precision results the analysis of the critical behaviour of full QCD, i.e in the presence of dynamical quarks, is still exploratory. Pisarski and Wilczek have argued that the chiral phase transition in QCD should be in the same universality class as three-dimensional chiral spin models with global $\operatorname{SU}\left(n_{\mathrm{f}}\right)$ $\times \mathrm{SU}\left(n_{\mathrm{f}}\right)$ symmetry [4]. Numerical simulations at intermediate gauge couplings seem to be in accordance with such a picture $[5,6]$.

First attempts have been undertaken to study the lattice size dependence of physical quantities [7]. A detailed analysis of critical exponents, however, has not been performed and, at least in the case of a continuous chiral transition, it would not profit from a finite-size scaling analysis as the correlation length is bounded due to the presence of finite quark masses. Besides the enormous numerical 
problems such an analysis has to face, there are at least two fundamental difficulties:

(i) In the staggered fermion formulation of QCD the $\operatorname{SU}\left(n_{\mathrm{f}}\right) \times \operatorname{SU}\left(n_{\mathrm{f}}\right)$ flavour symmetry is explicitly broken on the lattice and only gets restored in the continuum limit; the lattice action has only a global $\mathrm{U}(f) \times \mathrm{U}(f)$ symmetry, which due to the finite quark masses is explicitly broken to $\mathrm{U}(f)$. Only in the continuum limit will the full chiral symmetry be restored with $n_{\mathrm{f}}=4 f$ [8]. As the number of staggered fermion species $f$ is integer, it is even more complicated to understand in terms of the symmetries of the lattice lagrangian how the continuum symmetry group is approached in cases where the number of species is not a multiple of 4 . Of particular importance is the case of two flavours in the continuum limit, where the symmetry group is $S U(2) \times S U(2)$ and the critical exponents should be given by the corresponding three-dimensional $\mathrm{O}(4)$ exponents [9], if the transition is second order.

(ii) At least in the case where the chiral transition is continuous the standard finite-size scaling analysis, performed in pure gauge theories, is not applicable due to the presence of an explicit symmetry breaking field - the quark masses.

In this paper we want to address both of the above-mentioned points. We will study the chiral phase transition in strong coupling $\operatorname{QCD}(\beta=0)$ for one species of staggered fermions $(f=1)$. We will show that the transition is second order in this case, which is in accordance with results from mean-field calculations [10,11]. However, it is strikingly different from the first-order transition found in fourflavour QCD $\left(n_{\mathrm{f}}=4\right)$ at larger values of $\beta$ [5].

We will discuss the general strategy of determining critical exponents from lattice simulations in the presence of a symmetry breaking field (finite quark masses $m$ ) in the lagrangian. We concentrate on the determination of the exponent $\delta$, which controls the mass dependence of the order parameter at the critical point. We show that the ratio $m \chi /\langle\bar{\psi} \psi\rangle$ ( $\chi$ denoting the susceptibility) gives a direct estimate of the zero-mass critical coupling from measurements at finite quark masses and its value at this coupling is equal to the critical exponent $1 / \delta$. This ratio is a scaling function with properties similar to that of the familiar Binder cumulant for the order parameter used in finite-size scaling analysis of temperature-driven phase transitions [12]. We study this scaling function in mean-field as well as in a Monte Carlo simulation of the strong coupling theory. The latter shows that the critical behaviour is controlled by an effective theory which is in the same universality class as the three-dimensional $O(2)$ spin model, as one would expect, if this transition is controlled by the restoration of the chiral $U(1) \times U(1)$ symmetry of the lattice lagrangian.

This paper is organized as follows. In sect. 2 we outline the formulation of finite-temperature QCD in the strong coupling limit and discuss some new features of the monomer-dimer-polymer (MDP) algorithm used in our simulations $[13,14]$. In sect. 3 we discuss the general framework for the determination of 
critical exponents in the presence of finite quark masses and introduce some observables, which are particularly suited for lattice simulations of QCD. Sect. 4 contains a discussion of mean-field results for various observables defined in sect. 2 and the resulting critical exponents. Numerical results for strong coupling QCD are discussed in sect. 5. Finally we give our conclusions in sect. 6 where we will also discuss the relevance of the present analysis for future studies of the critical behaviour of two-flavour QCD.

\section{Strong coupling QCD at finite temperature}

In the strong coupling limit the QCD partition function is given by

$$
Z(m, \gamma)=\int \mathrm{d} \bar{\psi} \mathrm{d} \psi \int \mathrm{d} U \mathrm{e}^{S} .
$$

Here $\gamma$ denotes an additional coupling in the staggered fermion action, which in the continuum limit is related to the introduction of an anisotropy in the spatial and temporal lattice spacings [15]. The lattice action for staggered fermions with anisotropic couplings is then given by

$$
S=\sum_{n, m} \bar{\psi}_{n} Q_{n m} \psi_{m},
$$

with

$$
\begin{aligned}
Q_{n m} & =\sum_{\mu=1}^{3} D_{n m ; \mu}+\gamma D_{n, m ; 0}+m \delta_{n m}, \\
D_{n m ; \mu} & =\frac{1}{2} \eta_{\mu}(n)\left[U_{n, \mu} \delta_{n, m-\hat{\mu}}-U_{m, \mu}^{\dagger} \delta_{n, m+\hat{\mu}}\right] .
\end{aligned}
$$

At zero temperature chiral symmetry is broken in strong coupling QCD for any value of $\gamma$. A non-zero temperature is introduced through the finite extent of the lattice in the temporal direction $\left(N_{\tau} \ll N_{\sigma}\right)$. For fixed $N_{\tau}$ there exists a chiral symmetry restoring phase transition at some value $\gamma\left(N_{\tau}\right)$, with $\gamma\left(N_{\tau}\right) \rightarrow \infty$ for $N_{\tau} \rightarrow \infty$. We can vary the temperature of the system by varying the coupling $\gamma$ on lattices with fixed $N_{\tau}$. This is in complete analogy to the approach used at non-zero gauge coupling $\beta$. However, unlike in the weak coupling limit, $\beta \rightarrow \infty$, where the anisotropy parameter can directly be related to the temporal and spatial lattice spacings $\left(a_{\tau}\right.$ and $a_{\sigma}$ ) [16] and thus allows one to establish a connection between $\gamma$ and the temperature of the system, $T=1 / N_{\tau} a_{\tau}$, such a relation does not exist in the strong coupling limit. Also the generic choice, $\gamma=1$, which often is used in Monte Carlo simulations at non-zero gauge coupling $\beta$, is not applicable at $\beta=0$ as this would require a too small thermal extent of the lattice $\left(N_{\tau}<1\right)$ in 
order to reach temperatures high enough for a phase transition to occur. We thus will analyze the temperature dependence of strong coupling QCD by varying $\gamma$, keeping the temporal lattice size $N_{\tau}$ fixed. Throughout this paper we will use $N_{\tau}=4$.

The finite-temperature behaviour of strong coupling QCD has been studied in mean-field theory $[10,11]$ and also numerically, using the monomer-dimer-polymer (MDP) algorithm [13,14,17]. These studies suggest that the finite-temperature chiral phase transition is continuous for $\beta=0$. The MDP algorithm is particularly well suited to the analysis of strong coupling QCD as it allows a straightforward calculation of fermionic observables like the order parameter or the susceptibility. In the case of $\mathrm{SU}(N)$ gauge groups with $N$ being odd some care has to be taken in the implementation of the MDP algorithm in order to avoid the appearance of negative Boltzmann weights in the MDP partition function. The implementation of the algorithm for the SU(3) gauge group has been described in detail in ref. [14]. The only modification necessary for our simulations on anisotropic lattices is the proper mapping of baryonic loops onto monomer-dimer loops [14]. Some details of this mapping are described in appendix A.

We will concentrate in the following on a discussion of the chiral condensate, $\langle\bar{\psi} \psi\rangle$, and the susceptibility $\chi$. Both are easily calculated from the distribution of monomers on the lattice,

$$
\begin{aligned}
\langle\bar{\psi} \psi\rangle & =\frac{1}{N_{\sigma}^{3} N_{\tau}} \frac{\partial}{\partial m} \ln Z(m, \gamma) \\
& =\frac{1}{N_{\sigma}^{3} N_{\tau} m}\left\langle N_{m}\right\rangle \\
\chi & =\frac{\partial}{\partial m}\langle\bar{\psi} \psi\rangle \\
& =\frac{1}{N_{\sigma}^{3} N_{\tau} m^{2}}\left[\left(\left\langle N_{m}^{2}\right\rangle-\left\langle N_{m}\right\rangle^{2}\right)-\left\langle N_{m}\right\rangle\right] .
\end{aligned}
$$

\section{Critical exponents of finite-temperature QCD}

For vanishing quark masses the action for one species of staggered fermions, given in eqs. (2)-(4), is invariant under global $U(1) \times U(1)$ transformations,

$$
\begin{array}{lll}
\text { even: } & \chi^{\prime}=\mathrm{e}^{i \alpha} \chi, & \bar{\chi}^{\prime}=\mathrm{e}^{-i \beta} \bar{\chi}, \\
\text { odd }: & \chi^{\prime}=\mathrm{e}^{i \beta} \chi, & \bar{\chi}^{\prime}=\mathrm{e}^{-i \alpha} \bar{\chi} .
\end{array}
$$


This symmetry is spontaneously broken to the diagonal subroup: $U(1)_{\text {even }} \times U(1)_{\text {odd }}$ $\mapsto \mathrm{U}(1)$, due to the presence of a non-vanishing quark mass.

The mean-field studies [10,11] as well as the MDP simulation for SU(2) [17] suggest that the chiral phase transition in strong coupling QCD may be second order. In that case the transition will most likely only exist in the limit of vanishing quark mass and thermodynamic functions will not be singular, if the infinite-volume limit is taken at fixed, non-vanishing quark mass. For instance, the critical exponent $\delta$, describing the variation of the order parameter with quark mass is defined as

$$
O(m)=\lim _{N_{\sigma} \rightarrow \infty}\langle\bar{\psi} \psi\rangle\left(m, t, N_{\sigma}\right)_{t=0} \propto m^{1 / \delta}
$$

where

$$
t=\frac{\left(\gamma-\gamma_{0}\right)}{\gamma_{0}}
$$

denotes the reduced coupling and $N_{\sigma}$ denotes the spatial extent of the lattice. The susceptibility, $\chi(t)$, is given by

$$
\begin{aligned}
\chi(t) & =\lim _{m \rightarrow 0} \lim _{N_{\sigma} \rightarrow \infty} \chi\left(t, m, N_{\sigma}\right) \propto t^{-\gamma}, \\
\chi\left(t, m, N_{\sigma}\right) & =\frac{1}{N_{\sigma}^{3} N_{\tau}} \frac{\partial^{2} \ln Z}{\partial m^{2}} .
\end{aligned}
$$

Similarly other exponents are obtained by first taking the infinite-volume limit and then the zero quark mass limit.

In the infinite-volume limit response functions like the susceptibility will peak at a certain pseudo-critical coupling, which still depends on the quark mass. In order to discuss the scaling properties of this pseudo-critical coupling as well as the height of the peaks let us start with the generic scaling properties of the singular part of the free energy density of a statistical ensemble in the presence of an external symmetry breaking field (the quark mass),

$$
f(t, m)=b^{-1} f\left(b^{y_{t}} t, b^{y_{h}} m\right)
$$

where $b$ is an arbitrary scale factor, $y_{t}$ and $y_{h}$ are the thermal and magnetic critical exponents. Choosing the scale factor, $b=m^{-1 / y_{h}}$, we can write the free energy density as

$$
f(t, m)=M^{1 / y_{h}} f\left(t m^{-y_{t} / y_{h}}, 1\right) .
$$


From this we easily find the familiar scaling law for the order parameter at the zero-mass critical point, $t=0$, eq. (7), with $\delta=y_{h} /\left(1-y_{h}\right)$. From eq. (12) the order parameter, $\langle\bar{\psi} \psi\rangle$, as well as the susceptibility, $\chi(t, m)$ are obtained,

$$
\begin{aligned}
\langle\bar{\psi} \psi\rangle(t, m) & =m^{1 / \delta} F\left(t m^{-y_{t} / y_{h}}\right), \\
\chi(t, m) & =\frac{1}{\delta} m^{1 / \delta-1}\left(F\left(t m^{-y_{t} / y_{h}}\right)-\frac{y_{t}}{1-y_{h}} t m^{-y_{t} / y_{h}} F^{\prime}\left(t m^{-y_{t} / y_{h}}\right)\right),
\end{aligned}
$$

with scaling functions $F$ and $F^{\prime}$. We note that at finite quark mass the susceptibility has a peak at

$$
t_{m}=c m^{y_{t} / y_{h}}
$$

The critical exponent $\delta$ can thus be extracted from the mass dependence of $\langle\bar{\psi} \psi\rangle$ and $\chi$ at $t_{0} \equiv 0$ as well as on the pseudo-critical point $t_{m}$ defined by eq. (15).

Another useful relation emerges from the ratio

$$
\Delta(t, m)=m \chi /\langle\bar{\psi} \psi\rangle \text {. }
$$

It is obvious from eqs. (13) and (14) that $\Delta(0, m)$ gives directly $1 / \delta$. Furthermore the slope of $\Delta$ increases with decreasing $m$,

$$
\Delta^{\prime}(m)=\left.\frac{\partial \Delta}{\partial t}\right|_{t=0} \propto m^{-y_{t} / y_{h}} .
$$

Thus the ratio $\Delta$ shows a behaviour similar to that of the Binder cumulant $\left(\left\langle O^{4}\right\rangle /\left\langle O^{2}\right\rangle^{2}\right)$ usually used to determine the infinite-volume critical point in finite-size scaling studies: The functions $\Delta(t, m)$ will cross at the $m=0$ critical point if plotted as a function of $t$ for various values of $m$. In the $m \rightarrow 0$ limit we find

$$
\lim _{m \rightarrow 0} \Delta(t, m)= \begin{cases}1, & t>0 \\ 1 / \delta, & t=0 \\ 0, & t<0\end{cases}
$$

Of course, there will be corrections coming from the non-singular part of the free energy. An example for this behaviour is shown in sect. 4 where we discuss the mean-field results for $\Delta$ and other thermodynamic quantities in the strong coupling limit of QCD.

How important are finite-size corrections in the analysis of critical exponents in the presence of finite quark masses? The correlation length, which may diverge at the critical point, stays finite on a finite lattice and/or in the presence of a finite quark mass. Depending on the relative magnitude of these ordering effects one or 
TABLE 1

Critical exponents for three-dimensional spin models and mean-field theory

\begin{tabular}{lllllll}
\hline & \multicolumn{1}{c}{$\beta$} & \multicolumn{1}{c}{$\not$} & \multicolumn{1}{c}{$\underline{\underline{c}}$} & \multicolumn{1}{c}{$\underline{\delta}$} & \multicolumn{1}{c}{$\mathrm{b}$} \\
\hline $\mathrm{Z}(2)$ & $0.3258(44)$ & $\mathbf{1 . 2 3 9 0 ( 7 1 )}$ & $0.6289(8)$ & $4.803(73)$ & $2.49(2)$ \\
$\mathrm{O}(2)$ & $0.3510(2)$ & $\mathbf{1 . 3 2 0 0 ( 5 )}$ & $0.6740(3)$ & $4.755(6)$ & $2.48(2)$ \\
$\mathrm{O}(\infty)$ & 0.5 & 2 & 1 & 5 & 2.5 \\
$\mathrm{MF}$ & 0.5 & 1 & 0.5 & 3 & 3 \\
\hline
\end{tabular}

the other will limit the magnitude of the correlation length at the pseudo-critical point, where the susceptibility has a peak. The height of this peak itself is limited by these effects,

$$
\chi_{\text {peak }} \propto \begin{cases}m^{1 / \delta-1} & \text { for } L \rightarrow \infty \\ L^{\gamma / \nu} & \text { for } m \rightarrow 0 .\end{cases}
$$

We thus conclude that finite-size effects in the transition region are negligible as long as the quark masses are large enough. From eq. (19) we find as a condition

$$
m>\text { const. } \times L^{-b}, \quad b=\frac{\gamma \delta}{\nu(\delta-1)} .
$$

Using critical exponents for continous transitions in three-dimensional $Z(2)$ or $\mathrm{O}(N)$ models we expect that the exponent $b$ is of order 2 . This is also confirmed by the numerical data we are going to present in sect. 5. Some critical exponents for these models are given in table $1[18-20]$. For $Z(2)$ the value for $\delta$ is calculated using the hyperscaling relation $\delta=1+\gamma / \beta$.

\section{Mean-field results}

The chiral phase transition in strong coupling QCD has been studied extensively in a large- $d$ expansion [10,11]. This leads to a local lagrangian for an effective model of the chiral condensate $\sigma$, which has mean-field exponents in this approximation. This model describes the data well outside the critical region, however it clearly cannot reproduce the critical exponents of strong coupling QCD as long as one uses only the leading-order large- $d$ results. Here we will pay particular attention to the mass and $\gamma$ dependence of the function $\Delta$ defined in eq. (16).

The mean-field free energy is given by

$$
F_{\mathrm{MF}}=\frac{1}{4} N_{\tau} \sigma^{2}-\ln Z_{\mathrm{sS}}
$$




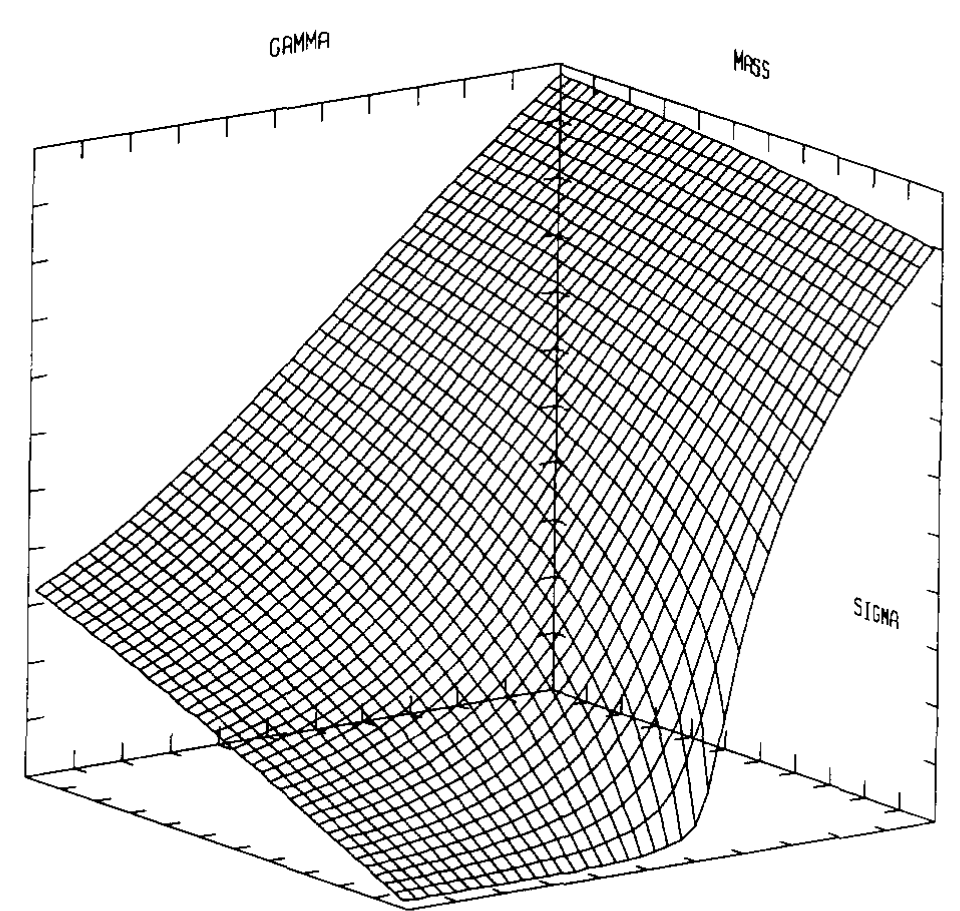

Fig 1. The mean-field chiral condensate $\sigma$ as a function of quark mass $m$ and coupling $\gamma$. The scale is given by the intervals $0 \leqslant m \leqslant 0.1,2.2 \leqslant \gamma \leqslant 3.2$ and $0 \leqslant \sigma \leqslant 1.2$.

where $Z_{\text {ss }}$ denotes the single-site partition function for SU(3) [10,11],

$$
Z_{\mathrm{ss}}=\frac{\sinh \left[4 N_{\tau} s\right]}{\sinh \left[N_{\tau} s\right]}+2
$$

with

$$
s \equiv \sinh ^{-1}[(\sigma+2 m) / 2 \gamma] \equiv \sinh ^{-1}(y)
$$

Minimizing the mean-field free energy for given values of $\gamma$ and $m$ produces the mean-field chiral condensate $\sigma$ (fig. 1). Consider this for small values of $y$. The condition for the minimum becomes

$$
-(m \gamma)+\left(\gamma^{2}-\frac{5}{3} N_{\tau}\right) y+\frac{1}{18} N_{\tau}\left(9 N_{\tau}^{2}-20\right) y^{3}+\mathrm{O}(y)^{5}=0
$$

Chiral symmetry is broken as long as the coefficient of the linear term is negative. The critical $\gamma$ is obtained by demanding that this coefficient vanishes,

$$
\gamma_{0}^{2}=\frac{5}{3} N_{\tau}
$$




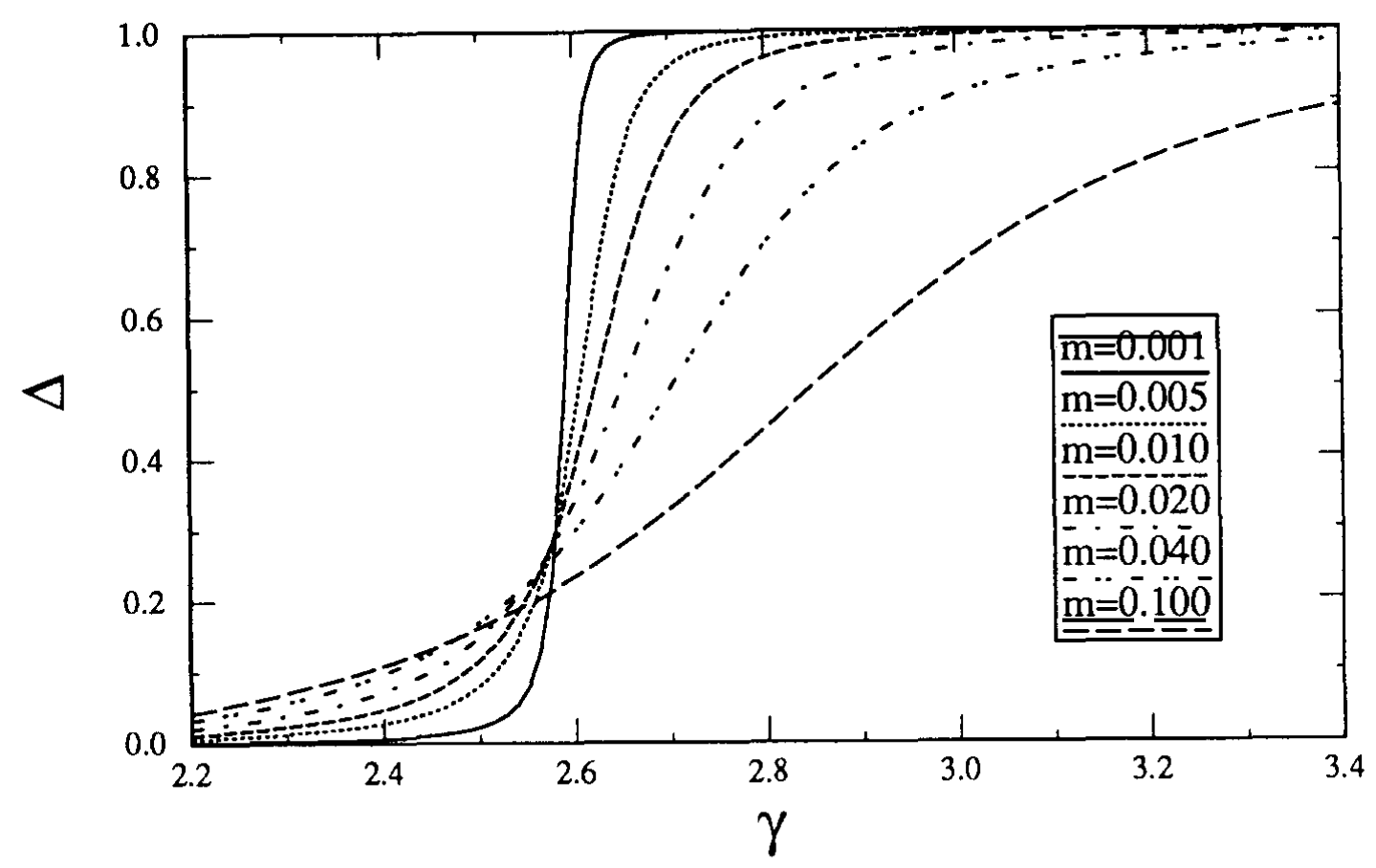

Fig. 2. Mean-field results for $\Delta(\gamma, m)$ as a function of $\gamma$ for various values of the quark mass.

Solving eq. (24) for $\sigma$ at the critical point $\gamma_{0}$ gives

$$
\sigma=2\left(\frac{50 N_{\tau} m}{9 N_{\tau}^{2}=20}\right)^{1 / 3}-2 m
$$

As expected we find that the critical exponent $\delta$ has the mean-field value $\delta=3$.

The ratio $\Delta$, defined in eq. (16), becomes

$$
\Delta\left(\gamma_{0}, m\right)=3\left[1+\left(\frac{4\left(9 N_{\tau}^{2}-20\right) m^{2}}{25 N_{\tau}}\right)^{1 / 3}+\mathrm{O}\left(m^{4 / 3}\right)\right]
$$

We note that with increasing $N_{\tau}$ the contributions from the non-singular part of the free energy gain increasing importance; the quark mass has to be reduced proportional to $1 / \sqrt{N_{\tau}}$ in order to keep the influence of these terms small. For $N_{\tau}=4$ and $m=0.005$ we find for instance, that $\Delta$ still deviates by $5 \%$ from its asymptotic value. In fig. 2 we show $\Delta(\gamma, m)$ as a function of $\gamma$ for various values of $m$. With decreasing mass $m$, subsequent curves give, indeed, a rather accurate estimate for $\gamma_{\mathrm{c}}$ as well as $1 / \delta$. Another useful way of presenting $\Delta$, which will be instructive in the analysis of numerical data is shown in fig. 3. This figure shows $\Delta$ as a function of $m$ for various couplings $\gamma$ in the vicinity of $\gamma_{0}$. This shows that the analysis of the behaviour of $\Delta$ in the limit $m \rightarrow 0$ yields a clear determination of 


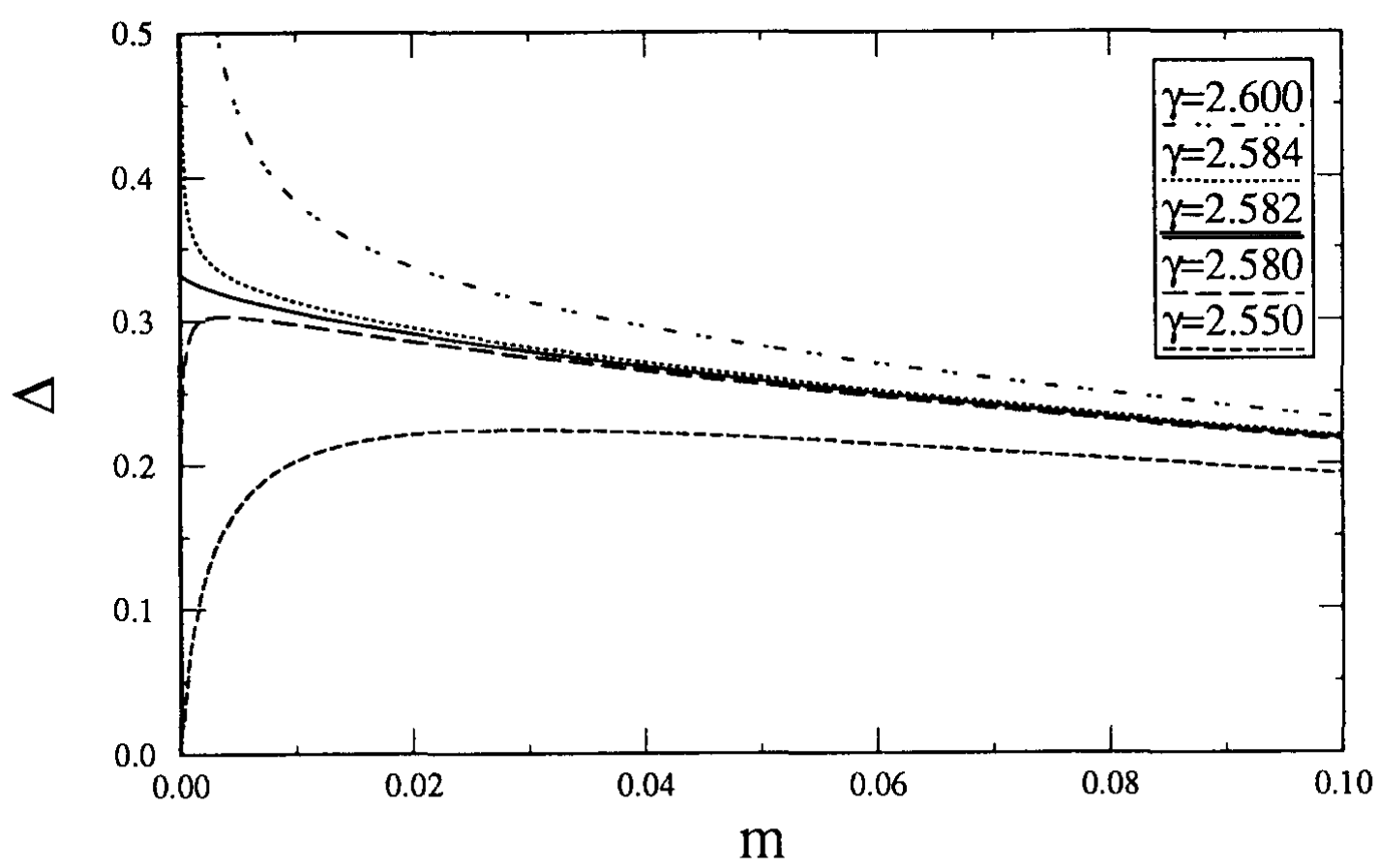

Fig. 3. Mean-field results for $\Delta$ as a function of the quark mass for various couplings $\gamma$ in the vicinity of $\gamma_{0}$.

$\gamma_{0}$. Moreover, we see that for fixed $\gamma<\gamma_{0}$ the maximum of $\Delta(\gamma, m)$ yields a rigorous upper bound for $\delta$.

\section{MDP simulation of strong coupling QCD}

By simulating the MDP system we measured the expectation value of the chiral condensate $\langle\bar{\psi} \psi\rangle$ and the susceptibility $\chi$ for strong coupling QCD on $N_{\sigma}^{3} \times N_{\tau}$ lattices with $N_{\sigma}=4,8,16$ and $N_{\tau}=4$. For the fermion mass $m$ we considered the values $m=0.005,0.01,0.02,0.04$ and 0.1 . From the recorded measurements of $\bar{\psi} \psi$ we constructed the corresponding distribution function and determined the integrated autocorrelation time $\tau_{\text {int }}(\bar{\psi} \psi)$ [21]. Typically we gathered $5 \times 10^{6} \mathrm{MDP}$ sweeps through the lattice, which for $N_{\sigma}=4$ and 8 corresponds to at least $2300 \tau_{\text {int }}$ measurements and for $N_{\sigma}=16$ to at least $300 \tau_{\text {int }}$ measurements. We discarded more than $20 \tau_{\text {int }}$ measurements for thermalization. Statistical errors for the chiral condensate were calculated using the integrated autocorrelation time [21]. The errors for the susceptibility were calculated using the jackknife method dividing the samples into eight blocks [22].

In fig. 4 we present the chiral condensate as a function of the fermion mass $m$ for various lattice sizes. It vanishes as the mass approaches zero showing the typical finite-size effects. The characteristics of an order parameter are only found by first taking the infinite-volume limit and then extrapolating to zero quark mass. 

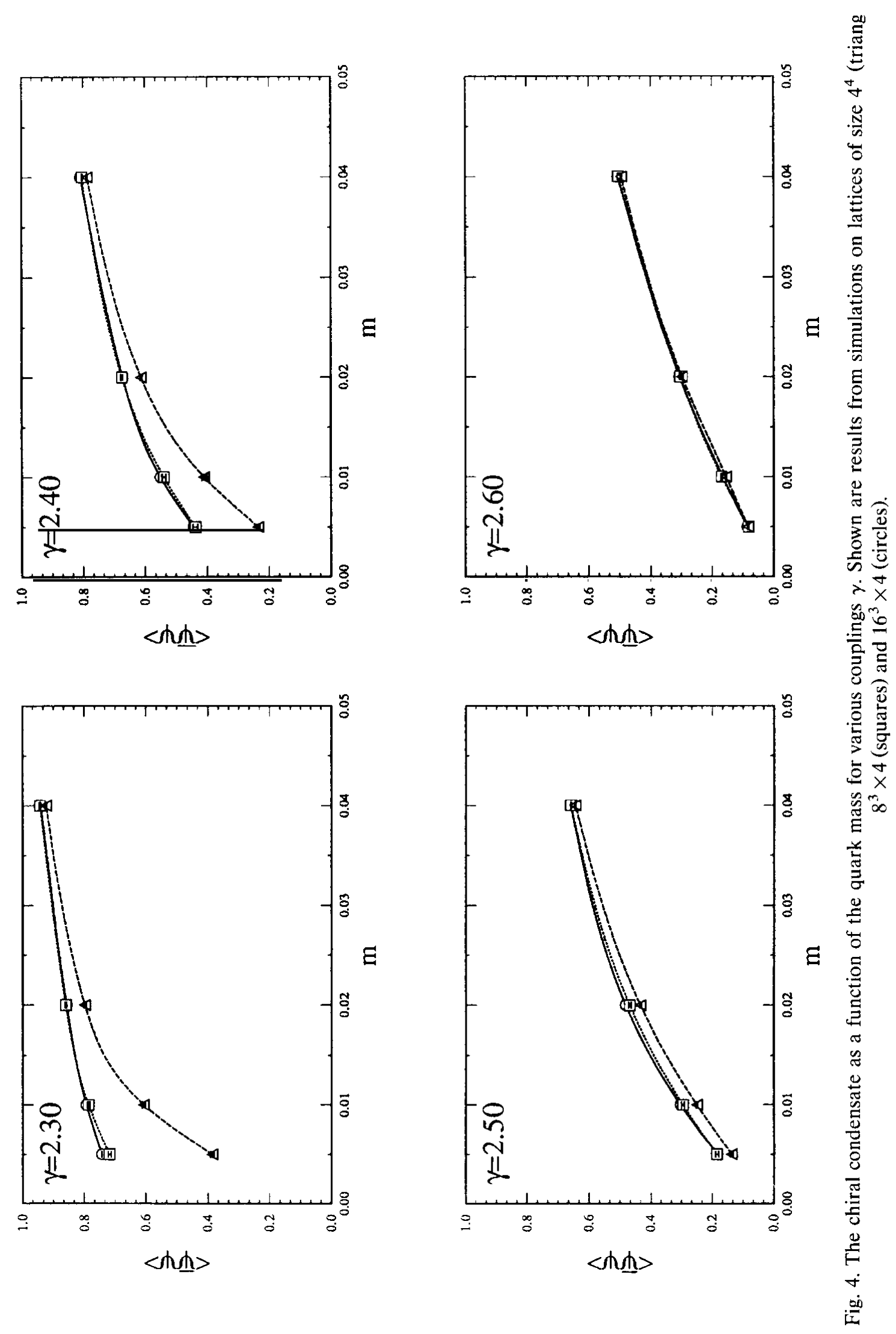

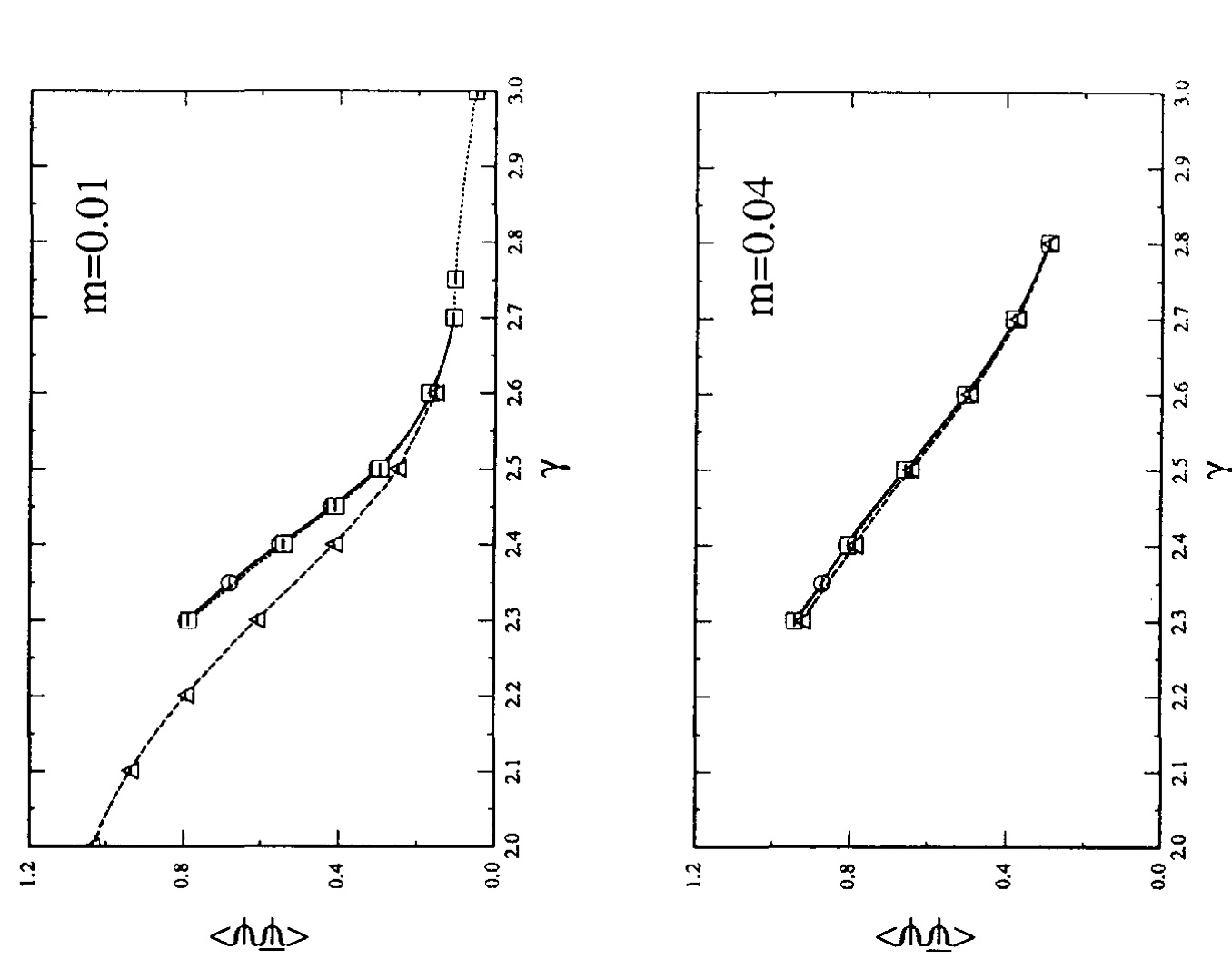

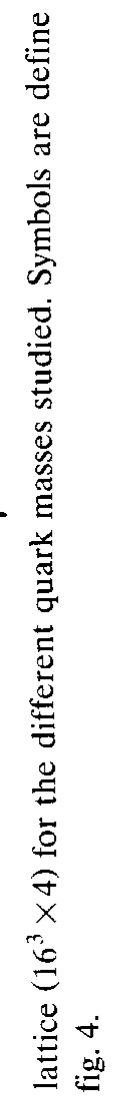
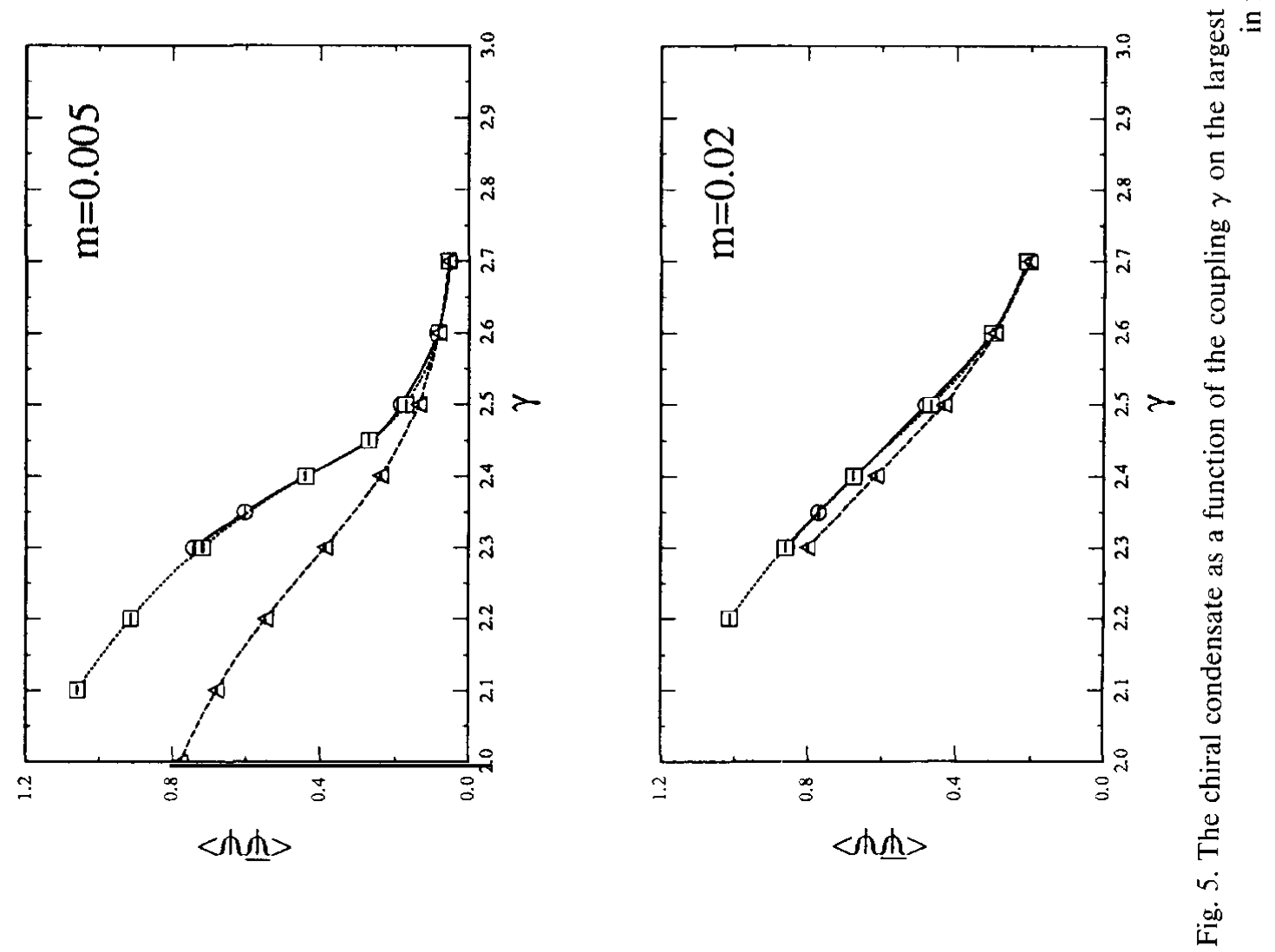


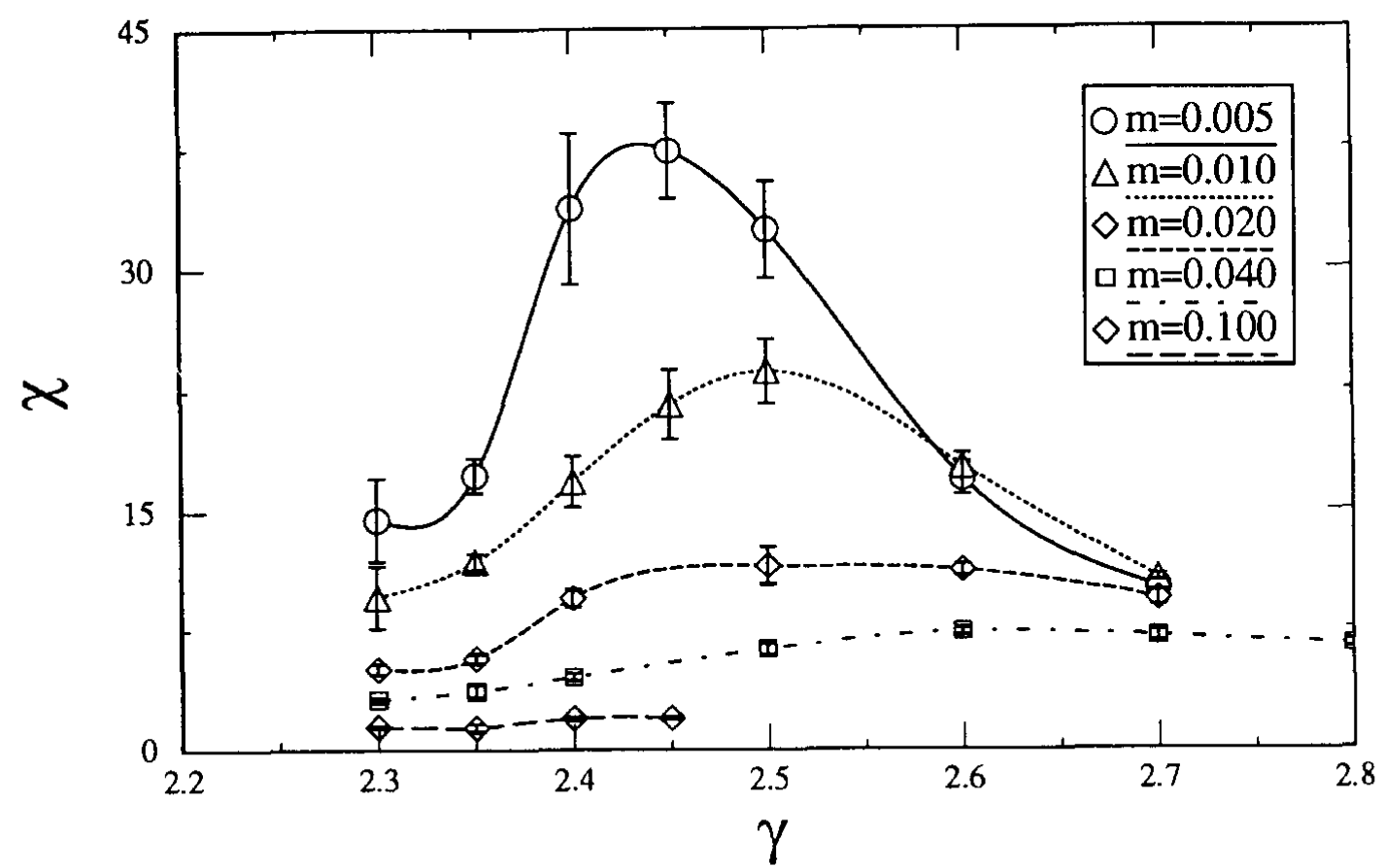

Fig. 6. The susceptibility $\chi$ as a function of the coupling $\gamma$ for $m=0.005,0.01,0.02,0.04$ and 0.1 for the $16^{3} \times 4$ lattice.

In particular in the critical region, i.e. for $\gamma=2.3-2.4$, we observe strong finite-size effects. We note that on the $4^{4}$ lattice these finite-size effects become negligible for $m \geqslant 0.05$, while on the $8^{3}$ lattice this is the case for $m \geqslant 0.01$. Such a shift of the finite-size effects to smaller masses is in accordance with eq. (19) with an exponent $b \simeq 2$. We thus expect finite-size effects to be negligible on our largest lattice in the entire mass range considered by us.

In fig. 5 we show the chiral condensate as a function of the anisotropy parameter $\gamma$ on our largest lattice, $\left(16^{3} \times 4\right)$, for the different quark masses studied. There is a smooth decrease of $\langle\bar{\psi} \psi\rangle$ as a function of $\gamma$ in the region $\gamma=2.3-2.4$ where we expect the phase transition. The function becomes steeper with decreasing quark mass $m$. For the quark masses used, none of the signals characteristic of a first-order phase transition were seen. The chiral condensate does not show a jump indicating a latent heat and no double peak structure in the distribution of $\bar{\psi} \psi$ is visible. Hence a first-order phase transition seems to be ruled out.

The susceptibility $\chi$ (fig. 6) shows a peak. The position $\gamma_{m}$ defines a pseudo-critical coupling which approaches the critical coupling $\gamma_{0}$ in the infinite-volume and zero-mass limit. The height of the maximum increases as the mass decreases, in agreement with the divergent behaviour of $\chi$ in that limit. As we concentrated in our simulations on the coupling regime close to $\gamma_{0}$, our statistics in the vicinity of the pseudo-critical couplings $\gamma_{m}$ is not sufficient to use the peak heights or their location for a precise determination of critical exponents (eqs. (14) and (15)). The 


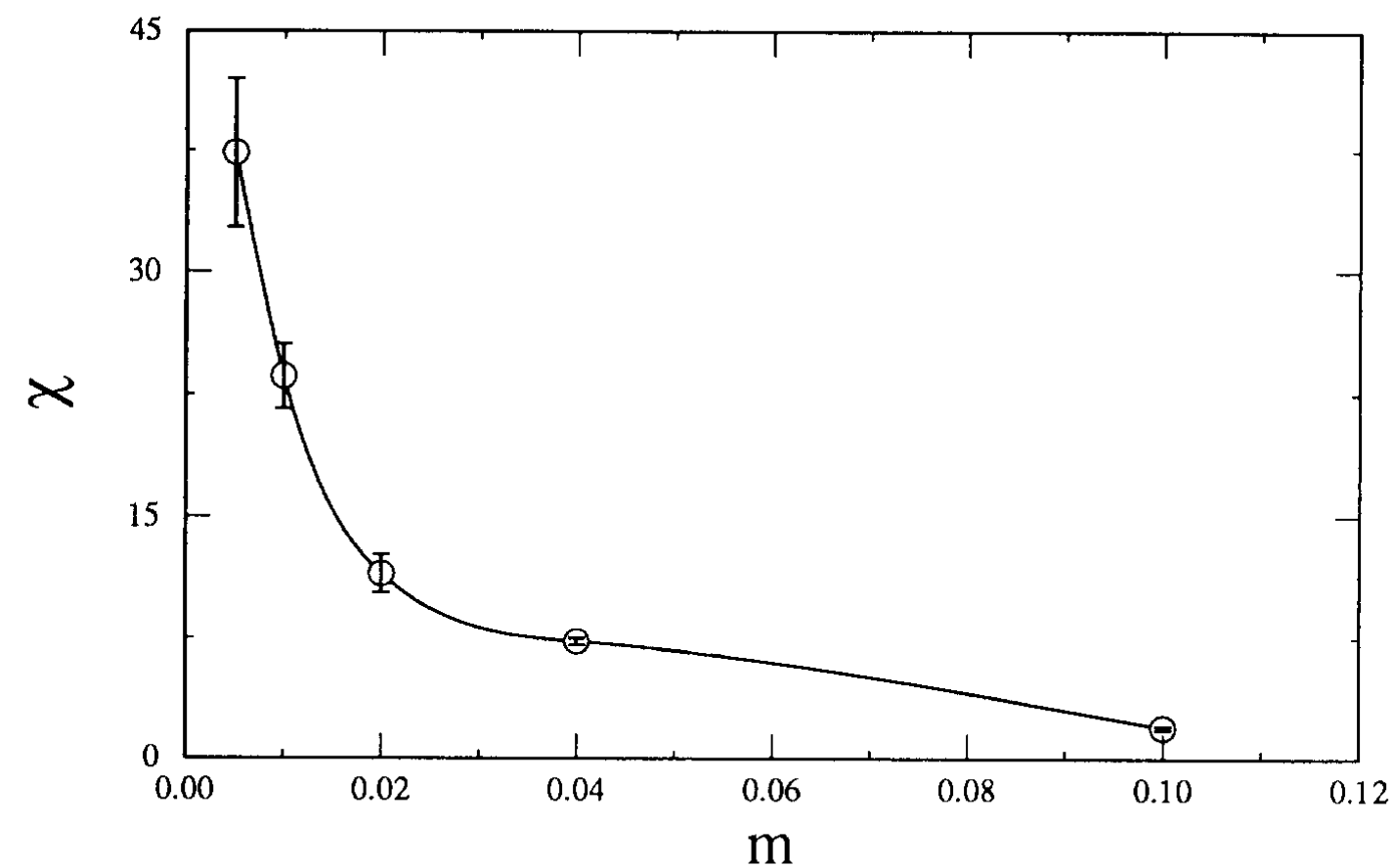

Fig. 7. Height of the peak of $\chi\left(\gamma_{m}, m\right)$ at the pseudo-critical coupling $\gamma_{m}$ as a function of the quark mass $m$.

general behaviour, however, is consistent with a second-order phase transition (fig. 7).

In the following we will determine the exponent $\delta$ from an analysis of the ratio $\Delta$, defined in eq. (16). This function is easily calculated in MDP simulations. In terms of the monomer distributions it is given by

$$
\Delta(\gamma, m)=\left[\frac{\left\langle N_{m}^{2}\right\rangle}{\left\langle N_{m}\right\rangle}-\left\langle N_{m}\right\rangle-1\right]
$$

In fig. 8 we show $\Delta$ as a function of $m$ for some values of $\gamma$. The general behaviour expected from eq. (18) and the mean-field solution shown in fig. 8 is clearly visible. From this we conclude that the critical coupling is in the interval

$$
2.35<\gamma_{\mathrm{c}}<2.4
$$

The mean-field analysis discussed in sect. 4 suggests that an upper bound on $\delta$ can be obtained from the maximum of $\Delta(\gamma, m)$ for any $\gamma<\gamma_{0}$, whereas a lower limit is obtained from a linear extrapolation in $m$ for $\gamma>\gamma_{0}$. However, while the upper bound is rigorous, we note that the lower limit can only be established for sufficiently large values of $\gamma$ as finite-mass correction to $\Delta(\gamma, 0)$ are proportional to $m^{y}$, with $y<1$. From the value of $\Delta$ obtained at $\gamma=2.35, m=0.01$ we extract 


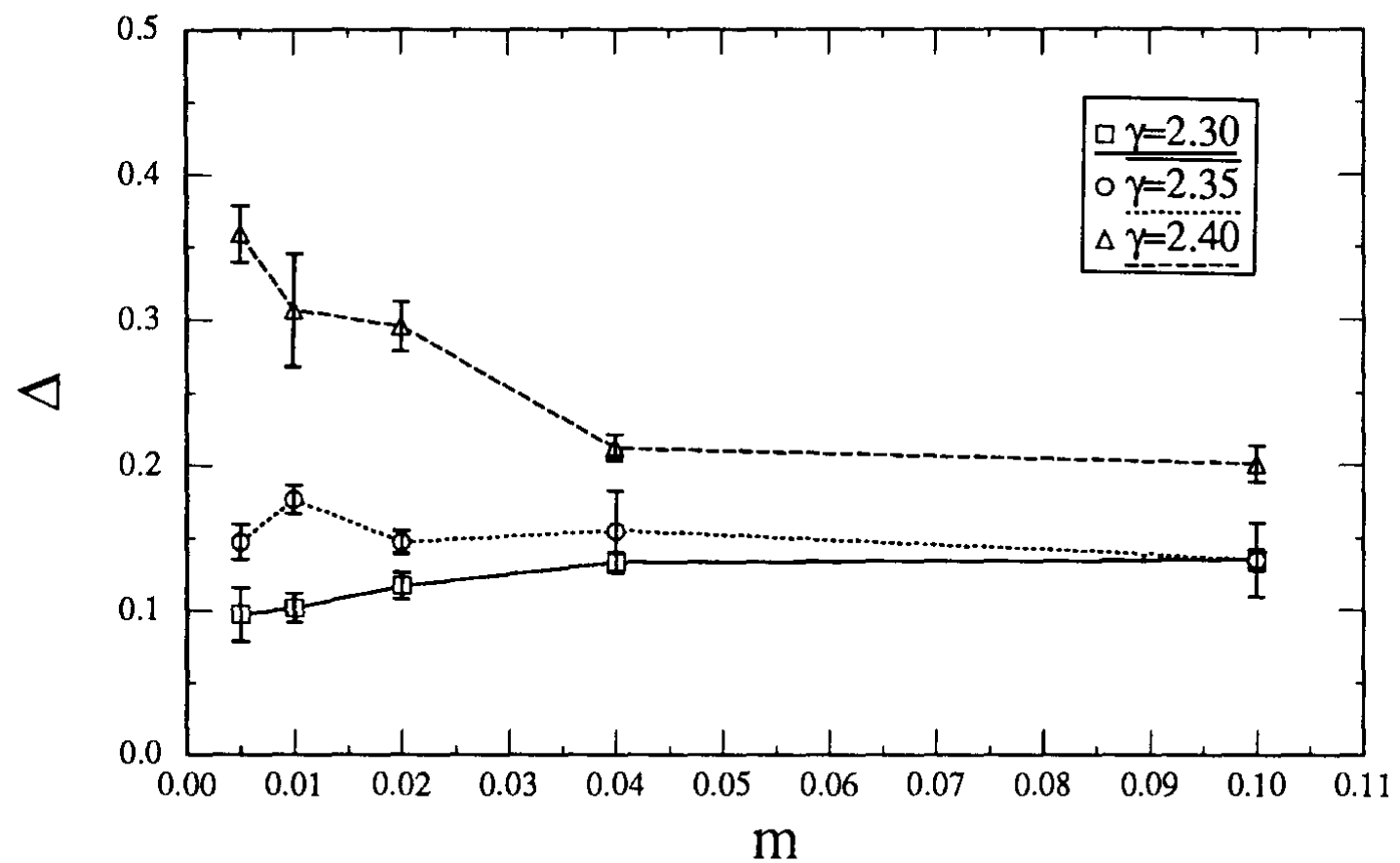

Fig. 8. $\Delta$ as a function of $m$ for $\gamma=2.30,2.35$ and 2.40 obtained from simulations on a $16^{3} \times 4$ lattice.

an upper limit on the critical exponent $\delta$, whereas a linear extrapolation of the data for $\gamma=2.4, m=0.04$ and 0.01 yields an estimate for the lower bound,

$$
0.18<1 / \delta<0.25 \text {. }
$$

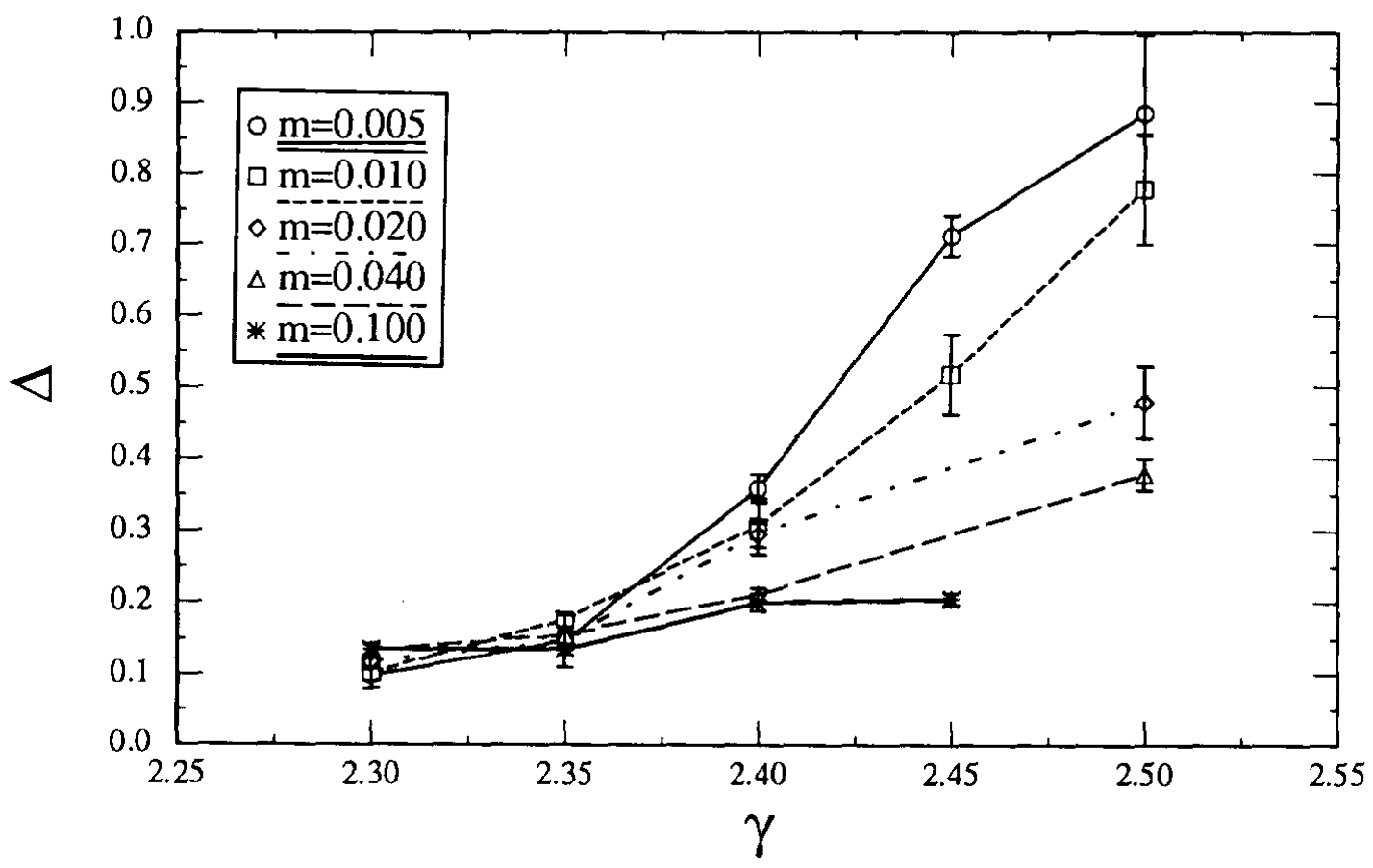

Fig. 9. $\Delta$ as a function of $\gamma$ for $m=0.005,0.01,0.02,0.04$ and 0.1 obtained from simulations on a $16^{3} \times 4$ lattice. 
These estimates are also supported by the $y$-dependence of $\Delta$ shown in fig. 9. This clearly shows that curves for different quark masses cross in the vicinity of $\gamma=2.35$. From our two smallest masses we find $\gamma_{0}=2.37 \pm 0.02$ and $1 / \delta=0.23 \pm$ 0.05 . This result for $\delta$ is in good agreement with the three-dimensional $\mathrm{O}(2)$ spin model predictions given in table 1 and it clearly differs from the mean-field value $\delta_{\mathrm{mf}}=3$.

\section{Conclusions}

We have presented a first analysis of the critical behaviour of QCD with light quarks in a case where the chiral phase transition is second order. It turned out that the ratio $m \chi /\langle\bar{\chi} \chi\rangle$ is particularly well suited for an analysis of the exponent $\delta$.

In strong coupling QCD with one species of staggered fermions the chiral transition turns out to be second order and the critical behaviour seems to be controlled by an $O(2)$ symmetric three-dimensional spin model. This is in accordance with the general arguments given by Pisarski and Wilczeck and clearly demonstrates the importance of the $U(1) \times U(1)$ symmetry of the staggered fermion lagrangian for the chiral phase transition. In the case of one species of staggered fermions the second-order phase transition of the strong coupling theory turns over into a first-order transition once the SU(4) $\times$ SU(4) symmetry of the continuum theory is sufficiently well approximated by the lattice theory. In the case of two-flavour QCD the approach to continuum QCD will be more subtle and, at present, it is not clear whether the continuous transition found in Monte Carlo simulations at finite gauge couplings is still controlled by the strong coupling symmetries of the lagrangian or does indeed reflect a second-order transition with $\mathrm{SU}(2) \times \mathrm{SU}(2) \simeq \mathrm{O}(4)$ exponents. A detailed determination of these exponents will be of great interest in the future.

\section{Appendix A}

THE MDP ALGORITHM

Writing the partition function in terms of monomers, dimers and baryonic loops results in the following representation [15]:

$$
\begin{aligned}
Z(m, \gamma) & =\sum_{\text {configurations }}(2 m)^{N_{m}} \prod_{\text {sites }} w(x) \prod_{\text {links }} w(x, y) \prod_{\text {loops }} \hat{w}(l), \\
\hat{w}(l) & =2 \gamma^{3 N_{\mathrm{t}}} \sigma(l),
\end{aligned}
$$




$$
\sigma(l)=(-1)^{k+N_{-}+1} \prod_{\langle x y\rangle \in l} \eta(x, y)
$$

In eqs. (A.1)-(A.3) $k$ denotes the winding number, $N_{\mathrm{t}}$ the number of links in the time direction, and $N_{-}$the total number of links in the negative space or time directions. The orientation of a loop, $\sigma(l)$, can either be +1 or -1 depending on $k, N_{-}$and the sign factors $\eta(x, y)$ associated with staggered fermions. This form of the baryonic loop weight is not yet suitable for a simulation since $\hat{w}(l)$ can be negative. A solution to this sign problem can be obtained by defining appropriate equivalence classes.

Each baryon loop is substituted by a chain of alternating single and double dimer lines along the path of the loop. This is called a polymer. For each baryon loop two possible polymers exist, one starting with a double dimer line and the other starting with a single dimer line. The summation over configurations is then done only over monomer dimer configurations. The weight of a baryonic loop $\hat{w}(l)$ is added to the weights $w_{1}(l)$ and $w_{2}(l)$ of the two dimer loops representing it in such a way that the total probability is the same, and the resulting loop weight $w(l)$ is non-negative.

$$
\begin{aligned}
& \sum_{--}^{--\urcorner} \quad \hat{w}(l)=2 \gamma^{3 N_{\mathrm{t}}} \sigma(l) \\
& =\frac{\gamma^{n}}{\gamma^{n}+\gamma^{-n}} 2 \gamma^{3 N_{\mathrm{t}}} \sigma(l)+\frac{\gamma^{-n}}{\gamma^{n}+\gamma^{-n}} 2 \gamma^{3 N_{\mathrm{t}}} \sigma(l) .
\end{aligned}
$$

The first term in eq. (A.2) is then added to the dimer loop carrying the weight $w_{1}(l)$ and the second term to the dimer loop with the weight $w_{2}(l)$.

$$
\begin{array}{ll}
{\left[\quad w_{1}(l)=\gamma^{2 N_{\mathrm{Dt}}}+\frac{\gamma^{n}}{\gamma^{n}+\gamma^{-n}} 2 \gamma^{3 N_{\mathrm{t}}} \sigma(l),\right.} \\
w_{-\infty} w_{2}(l)=\gamma^{2 N_{\mathrm{Dt}}+\frac{\gamma^{-n}}{\gamma^{n}+\gamma^{-n}} 2 \gamma^{3 N_{\mathrm{t}}} \sigma(l) .}
\end{array}
$$

For the first loop the variable $n$ takes the value $n=-n_{\mathrm{t}}$. For the second loop the sign of $n$ changes and $n=+n_{\mathrm{t}}$. The resulting new loop weight $w(l)$ takes its minimum value $w(l)=0$ when $\sigma(l)$ is minus one and $n_{\mathrm{t}}$ equals zero. In all other possible cases $w(l)$ is strictly positive,

$$
w(l)=\gamma^{2 N_{\mathrm{Dt}}}\left(1+\frac{2 \sigma(l)}{\gamma^{n_{\mathrm{t}}}+\gamma^{-n_{\mathrm{t}}}}\right) .
$$


In eqs. (A.5)-(A.7) $N_{\mathrm{Dt}}$ is the total number of dimer lines in the time direction. The quantity $n_{\mathrm{t}}=3 N_{\mathrm{t}}-2 N_{\mathrm{Dt}}$ gives the difference between the number of single and double dimer lines in the time direction.

The algorithm used in our Monte Carlo simulation is based on the size and link weights $w(x)$ and $w(x, y)$ as defined in ref. [15] with a new loop weight $w(l) \geqslant 0$ given by eq. (A.7).

The actual update procedure is based on the observation that any valid MDP configuration can be mapped on a configuration consisting exclusively of monomers. Thus a sufficient step is a change from two neighbouring monomers to a dimer-line or vice versa. However at small values of the fermion mass this results in a large autocorrelation time since the probability of creating a monomer is proportional to the fermion mass. By implementing a plaquette-update scheme which does not involve the creation of monomers we could improve the efficienty of our MPD algorithm. This plaquette-update is characterized by two properties. It has the ability to change between timelike and spacelike dimer lines and it is able to move a monomer by two lattice spacings. The new update scheme is independant of the mass and therefore allows us to sample the phase space more efficiently at small values of the fermion mass $m$. We find that this update scheme strongly reduces the autocorrelation time of operators depending on the number of monomers.

\section{References}

[1] A. Ukawa, Nucl. Phys. B (Proc. Suppl.) 17 (1990) 118

[2] B. Svetitsky and G. Yaffe, Nucl. Phys. B210 (1982) 423

[3] J. Engels, J. Fingberg and M. Weber, Nucl. Phys. B332 (1990) 737

[4] R.D. Pisarski and F. Wilczek, Phys. Rev. D29 (1984) 338

[5] R.V. Gavai, S. Gupta, A. Irbäck, F. Karsch, S. Meyer, B. Petersson, H. Satz and H.W. Wyld, Phys. Lett. B241 (1990) 567

[6] F.R. Brown, F.P. Butler, H. Chen, N.H. Christ, Z. Dong, W. Schaffer, L.I. Unger and A. Vaccarino, Phys. Lett. B251 (1990) 181; Phys. Rev. Lett. 65 (1990) 2491

[7] M. Fukugita, H. Mino, M. Okawa and A. Ukawa, Phys. Rev. Lett. 65 (1990) 816

[8] H. Kluberg-Stern, A. Morel, O. Napoly and B. Petersson, Nucl. Phys. B220 (1983) 447

[9] K. Jansen, F. Karsch, T. Neuhaus and B. Plache, Critical behaviour of the O(4) nonlinear $\sigma$-model in 3 and $3+1$ dimensions, in preparation

[10] P.H. Damgaard, N. Kawamoto and K. Shigemoto, Nucl. Phys. B264 (1986) 1

[11] G. Fäldt and B. Petersson, Nucl. Phys. B265 (1986) 197

[12] K. Binder, Z. Phys. B43 (1981) 119

[13] P. Rossi and U. Wolff, Nucl. Phys. B248 (1984) 105

[14] F. Karsch and K.-H. Mütter, Nucl. Phys. B313 (1989) 541

[15] F. Karsch, Nucl. Phys. B205 [FS5] (1982) 285

[16] F. Karsch and I.O. Stamatescu, Phys. Lett. B227 (1989) 153

[17] J.-U. Klaetke and K.-H. Mütter, Nucl. Phys. B342 (1990) 764

[18] A.M. Ferrenberg and D.P. Landau, Phys. Rev. B44 (1991) 5081

[19] D.J. Wallace, in Phase transitions and critical phenomena, Vol. 6, ed. C. Domb and J.L. Lebovitz (Academic Press, New York, 1983) p. 293 
[20] S. Ma, in Phase transitions and critical phenomena, Vol. 6, ed. C Domb and J.L. Lobovitz (Academic Press, New York, 1983) p. 249

[21] A.D. Sokal, Monte Carlo methods in statistical mechanics: Foundations and new algorithms, lecture notes "Cours de Troisième Cycle de la Physique en Suisse Romande", Lausanne 1989

[22] R.G. Miller, Biometrica 61 (1974) 1 